\title{
La investigación como método de expresión cultural en la IED Anaxímenes Torres Ospino, Magdalena ${ }^{1}$ Research as a method of cultural expression in the IED Anaximenes Torres Ospino, Magdalena
}

DOI: http://dx.doi.org/10.17981/cultedusoc.9.3.2018.72

Artículo de investigación. Fecha de recepción: 15/06/2018. Fecha de aceptación: 27/11/2018

Friceth Quintero-Sánchez"2;

Faride Araque-Castro y Jorge Córdoba-Arboleda ${ }^{3}$

Institución Educativa Departamental Anaximenes Torres Ospino (Colombia)

Para citar este artículo:

Quintero-Sánchez, F., Araque-Castro, F. y Córdoba-Arboleda, J. (2018). La investigación como método de expresión cultural en la IED Anaxímenes Torres Ospino, Magdalena. Cultura. Educación y Sociedad 9(3), 613-618. DOI: http://dx.doi.org/10.17981/cultedusoc.9.3.2018.72

\section{Resumen}

En los últimos años se ha evidenciado un cambio negativo en el municipio de El Banco, Magdalena, pues los habitantes han perdido interés por la cultura y patrimonio que hace parte de la historia e identidad del municipio. Lo mencionado anteriormente da origen al proyecto de investigación que es objeto de este artículo, el cual tiene por objetivo volver a inculcar el sentido de pertenencia en los estudiantes de la Institución Educativa Departamental Anaxímenes Torres Ospino, lo que va a permitir que se cuide y mantenga de manera prolongada el patrimonio y la cultura del sitio. Su metodología se realizó bajo un enfoque cualitativo, utilizando el tipo de investigación acción, desde un diseño descriptivo, exploratorio. Para la recolección de datos se utilizaron técnicas como; observación participante y diario de campo. Se llevó a cabo un muestreo intencional o de conveniencia, escogiendo los estudiantes que tenían especial interés en temas relacionados con la cultura. La muestra estuvo conformada por ciento treinta (130) estudiantes con edades que oscilaron entre los cuatro (4) y doce (12) años, de grados comprendidos entre sexto y décimo de la IED Anaxímenes Torres Ospino, Magdalena. Los resultados demuestran que con los modelos de educación tradicional no se hace énfasis en la cultura propia del municipio, teniendo como consecuencia un poco o nulo interés de los estudiantes en conocer la cultura interna de ellos, pero al momento de ser implementada la Investigación y la utilización de las TIC en el aula, se fortalecen los deseos de búsqueda y conocimientos hacia su propia historia y cultura.

Palabras clave: Investigación, cultura, cuidado, patrimonio, historia.

\section{Abstract}

In recent years there has been a negative change in the municipality of El Banco, Magdalena, as the inhabitants have lost interest in the culture and heritage that is part of the history and identity of the municipality. The aforementioned gives rise to the research project that is the subject of this article, which aims to re-instill the sense of belonging in the students of the Anaximenes Torres Ospino Departmental Educational Institution, which will allow for care and maintenance in a prolonged way the patrimony and the culture of the site. Its methodology was carried out under a qualitative approach, using the type of action research, from a descriptive, exploratory design. For the collection of data, techniques such as; participant observation and field diary. An intentional or convenience sampling was carried out, choosing the students who had special interest in topics related to culture. The sample consisted of one hundred and thirty (130) students with ages ranging between four (4) and twelve (12) years, of grades between sixth and tenth of the IED Anaximenes Torres Ospino, Magdalena. The results show that with the traditional education models there is no emphasis on the culture of the municipality, having as a consequence a little or no interest of the students in knowing the internal culture of them, but at the moment of being implemented the Research and the Using ICT in the classroom, the search and knowledge desires are strengthened towards their own history and culture.

Keywords: Research, culture, care, heritage, history.

1 Este artículo ha sido derivado del Programa de Fortalecimiento de la Cultura Ciudadana y Democrática CT+I a través de la IEP apoyada en TIC en el Departamento de Magdalena: CICLON

2 Líder del grupo de investigación "Soy Cultura".

3 Docentes de la Institución Educativa Departamental Anaximenes Torres Ospino y del grupo de investigación "Soy Cultura".

- The author; licensee Universidad de la Costa - CUC.

Cultura, Educación y Sociedad vol. 9 no. 3, pp. 613-618. Diciembre, 2018

Barranquilla. ISSN 2389-7724 Online 


\section{Introducción}

Todos los pueblos en el mundo, desde su surgimiento, crean y sostienen una cultura específica que impacta directamente en sus formas de vida diaria, creencias espirituales, filosofía y la forma en cómo se organizan las sociedades, las leyes éticas y jurídicas, la economía, la ciencia, el arte, su lenguaje, el tipo de educación, la historia de ellos propiamente dicha, entre otros (Díaz, 1996).

Para Zárate (1986) la pertenencia social como cultural son elementos fundamentales en una comunicación, siendo estos agrupados y enseñados en las aulas de clases para así, a través de la práctica de valores propios y conocimientos internos dentro de un pueblo es la principal forma en que este adquiere su identidad cultural.

\section{¿Qué se entiende por cultura?}

Todo lo existente en el mundo, es considerado cultura, siendo producida por la mente y la mano humana. Como lo es el caso de las fiestas, los alimentos, las formas de gobierno, pensar, el tipo de vestimenta y las modas (Krebs, 1999). En síntesis, todo aquello que sin importar su tipo material o inmaterial (pensamientos, valores, tipos de comportamiento y objetos específicos), que lleguen a identificar a un grupo específico de personas, y que haya sido adquirido por experiencia propia o conocimientos aprendidos, es considerado cultura (Barañano, 2011). En otras palabras, la manera en que los humanos desarrollan su vida, el lugar en donde habitan y cómo construyen su día a día es cultura, siendo la cultura un desarrollo, propiamente dicho, la civilización misma (Jiménez, 2008).

Al darse la yuxtaposición entre los idiomas y las culturas, los individuos progresan hasta adquirir una interculturalidad globalizada, (Almeida, 2007) así también los pueblos mejoran sus niveles organiza- cionales de aldeas a ciudades, alcanzando por último una megapolis (Alarcón, 2002).

\section{Concepto de Cultura según diversas Ciencias}

La cultura es mirada desde diferentes perspectivas de una manera en particular. Para la antropología la cultura es como ese conjunto de elementos organizados de una forma coherente y lógica de orden material o espiritual que componen una sociedad. (Claro, 2010). Ya para la sociología, la cultura es tomada como esos estímulos ambientales que en conjunto desarrollan la socialización de un individuo. (Salomón, 1980). En cambio para la filosofía, son el conjunto de resultados novedosos que el hombre tiene para transformar su entorno, repercutiendo del mismo modo en el propio individuo (García, 1996). Pero cuando se habla ya de una cultura general, se hace referencia a ese sistema de creencias y conocimientos que se le exigen a una persona para poder estar en un espacio y tiempo determinado según la sociedad en la que este se encuentre inmerso (García, 1996).

\section{Grupos étnicos e Identidad Cultural}

Como razón fundamental de conocer la diversidad de grupos étnicos existentes, es que la población está formada precisamente por ellos (Yúdice, 2002).

Entre los grupos étnicos existen diferencias a veces bastante marcadas por el idioma, el vestido y la composición social, así como su religión.

La Etnia es considera como esa agrupación natural compuesta por individuos pertenecientes a esa misma cultura que permite grupos raciales diversos (García, 1999). Siendo considerada la etnología como la ciencia que se encarga esencialmente del estudio de las razas y pueblos. La etnología busca dar respuesta a la cultura determinada por un pueblo (Sassoon, 2006). 


\section{El Idioma como pilar de la Cultura}

El idioma es para la lingüística ese tipo de lengua de un determinado país. El concepto de idioma inicia en el momento que una comunidad posee una lengua distinta a las demás y es consciente de ello, siendo un modo particular de comunicación de un grupo específico de humanos.

El idioma es un eje central al momento de construir sus cimientos una nueva cultura, siendo en particular el modo en que sus conocimientos, creencias y valores culturales son transmitidos en los pueblos (Batalla, 1997).

Un idioma es esa forma oral de expresión del ser humano, teniendo una destacada connotación en múltiples pueblos y regiones en todo el mundo. La diversidad de idiomas enriquece el conocimiento cultural y los prepara a las personas para salir al mundo. Haciendo necesaria la comprensión y entendimiento de la cultura y la forma en como esta ve la vida desde todos los ángulos, haciendo beneficiosa la comunicación entre pueblos (Camarero, 2004).

\section{La Interculturalidad Como Meta}

La Interculturalidad es esa convivencia tranquila que se da entre culturas; siendo la cooperación entre estas una fuerza para dar una colaboración y solidaridad entre ellas. Es esa relación justa y equitativa que se lleva a cabo en medio de culturas diversas (Pardo, 2003). Por este motivo se hace fundamental la enseñanza de cooperación y conocimientos de las culturas, creando una sana convivencia entre estas y educando acerca de cómo la importancia de la diversidad cultural es lo que mantiene a la sociedad en la posición actual, destacando que la prevalencia de paz entre culturas es un precursor de prosperidad y desarrollo.

\section{Metodología}

Se realizó un estudio de tipo cualitativo, utilizando el modelo de investigación acción, desde un diseño descriptivo, exploratorio. La investigación cualitativa busca conocer e interpretar la realidad de los participantes a través de sus propias experiencias, entregando una información subjetiva del fenómeno de estudio (Hernández, Fernández y Baptista, 2010). Por su parte el modelo de investigación acción plantea una producción de conocimiento basado en la reflexión de los participantes, teniendo en cuenta la participación activa de maestros en el proceso de enseñanza y acompañamiento tecnológico, guiando al estudiante en su proceso de investigación. Generando nuevo saber y conocimiento sobre una realidad determinada en un proceso de aprendizaje colaborativo.

Los estudios con un diseño descriptivo están encaminados a especificar las características de la población sujeto de estudio o los fenómenos que sean sometidos a análisis. Bajo un estudio de tipo exploratorio, debido a que no se han realizado estudios previos en la institución (Hernández, Fernández y Baptista, 2010). En cuanto a la población beneficiada con el estudio, se tomó una muestra representativa de ciento treinta (130) estudiantes de la IED Anaxímenes Torres Ospino, Magdalena, distribuidos en los cursos desde preescolar hasta quinto de primaria, con edades que oscilaron entre los doce (12) y dieciséis (16) años, se realizó un muestreo de tipo intencional, escogiendo los estudiantes que mostraban interés por el tema.

La metodología del proyecto de investigación se realizó a partir de los recorridos de las trayectorias de indagación, basados en la integración de la IEP apoyada en TIC al aula, articulado al plan de estudios, transversalizando así los 
procesos de lectura y escritura a todas las áreas del conocimiento, lo anterior con la finalidad de generar una trasformación social y educativa a través del aprendizaje en contexto, involucrando intereses, motivaciones y realidades de los estudiantes para generar conocimiento científico, donde se establecieron cinco (5) trayectos que especifican la ejecución y las metas trazadas para darle solución a la pregunta problema planteada. Los trayectos utilizados se presentan a continuación en la tabla 1.

\section{TABLA 1 \\ Proyectos}

\begin{tabular}{ll}
\hline & Los docentes realizaron \\
& un diagnóstico, según los \\
criterios de evaluación & de las pruebas por \\
Trayecto (1) & competencias para \\
& identificar las dificultades \\
& específicas que presentaba \\
& la población. \\
& Diseño de estrategias \\
& pedagógicas basadas en \\
& la investigación y las \\
TIC transversales a las \\
diferentes áreas de estudio, \\
de acuerdo a las necesidades \\
de la población. \\
Implementación y \\
transversalización de las \\
estrategias en el aula de \\
clase por parte del cuerpo \\
docente.
\end{tabular}

Fuente: elaboración propia.

\section{Resultados}

Luego de la realización de las actividades descritas anteriormente, los estudiantes relacionaban de manera correcta los conocimientos que adquirieron durante la fase teórica, fueron puestos en práctica durante la fase de danzas y sustentaciones, y se obtuvieron muy buenos resultados posteriores a la implementación de estas fases del proyecto, pues al final, los estudiantes identificaban de manera correcta los componentes históricos, culturales y patrimoniales del municipio de El Banco y del departamento del Magdalena. dándoles la iniciativa clara de cuidar y respetar ese ambiente, así mismo los hizo más conscientes de la importancia que tiene para el desarrollo no solo de ellos sino también de sus familias y el municipio en general, conocer sus riquezas no solo económicas sino también culturales que los fortalecen y hacen destacar. Un elemento a resaltar es cómo estos al recibir conocimientos acerca de sus cultura, historia y patrimonios propios del municipio, también impartieron estos conocimientos con las personas que tenían en su alrededor, mostrando que la consciencia sobre estos aspectos no solo estaba siendo acrecentada en los estudiantes, sino también, en la comunidad en general que entraba en contacto con ellos.

\section{Conclusiones}

La cultura es un aspecto muy importante de la historia de un lugar, es por esto que es necesario implementar proyectos que promuevan a que las personas se apropien, acepten, quieran y cuiden este elemento que representa de manera muy emotiva los lugares en el mundo. Mostrando como el enseñar la historia local y resaltar sus riquezas culturales, ayuda al cuidado de las mismas, pues se hacen 
más evidentes las razones por las cuales estas son importantes para su comunidad, mejorando y respetando las tradiciones históricas que ya están instauradas en determinado territorio. También nos hace reflexionar si realmente se están tomando las medidas necesarias para promover este tipo de proyecto en todas las comunidades.

\section{Referencias}

Alarcón, L., Conde, J. y Santos, A. (2002). Educación y cultura en el Estado soberano del Magdalena, 1857-1886. Barranquilla: Universidad del Atlántico.

Almeida-Leñero, L., Nava, M., Ramos, A., Espinosa, M., Ordoñez, M. y Jujnovsky, J. (2007). Servicios ecosistémicos en la cuenca del río Magdalena, Distrito Federal, México. Gaceta ecológica, (84-85). 117-123.

Barañano, N., Garate, J. y Bobowik, M. (2011). Valores y actitudes: cultura de violencia y paz. En, D, Paéz (Coord.), Superando la violencia colectiva $y$ construyendo una cultura de paz. (71104). España: Fundamentos.

Batalla, G. (1997). Nuestro patrimonio cultural: un laberinto de significados. El patrimonio nacional de México. México, D.F.: FCE-Conaculta.

Camarero, C. y Garrido, M. (2004). Marketing del patrimonio cultural. Madrid: Pirámide.

Claro, M. (2010). Impacto de las TIC en los aprendizajes de los estudiantes: estado del arte. Santiago de Chile: Cepal.

Díaz-Granados, M. (1996). Geografía económica del Magdalena Grande: 1946 1955. Santa Marta: Instituto de Cultura del Magdalena.

Durham, E. (2014). Cultura, patrimonio, preservación. Alteridades, (16), 131136.
García, J. (1998). De la cultura como patrimonio al patrimonio cultural. Política y sociedad, 27, 9.

García, M. (1996). Ciencia tecnología y sociedad. Cuadernos de iberoamerica. OEI.

García, N. y Criado, A. (1999). Los usos sociales del patrimonio cultural. Consejería de Cultura. En, Junta de Andalucía(Coord.). Patrimonioetnológico: nuevas perspectivas de estudio. (1633). Granada: Instituto Andaluz del Patrimonio Histórico

Jiménez Ramírez, M. (2008). Aproximación teórica de la exclusión social: complejidad e imprecisión del término. Consecuencias para el ámbito educativo. Estudios pedagógicos (Valdivia), 34(1), 173-186.

Krebs, M. y Schmidt-Hebbel, K. (1999). Patrimonio cultural: aspectos económicos y políticas de protección. Perspectivas de política, economíay gestión, 2, 207-245.

Mena, M. (2000). La América colonial, 1492-1763: cultura y vida cotidiana. Editorial Síntesis.

Molano, L. y Lucía, O. (2007). Identidad cultural un concepto que evoluciona. Revista Opera, (7).

Ospino, W. (1995). Las mujeres del Magdalena en la Guerra de Independencia de España (Vol. 6). Santa Marta: Instituto de Cultura del Magdalena.

Pardo, M. y Gómez, E. (2003). Etnobotánica: aprovechamiento tradicional de plantas y patrimonio cultural. Anales del Jardín Botánico de Madrid, 60,(1). 171-182.

Pinto, M. (1986). La identidad cultural de la vanguardia en Latinoamérica. En, Identidad cultural de Iberoamérica en su literatura. (102-110). Madrid: Editorial Alhambra. 
Reichel-Dolmatoff, G. (1951). Datos histórico-culturales sobre las tribus de la antigua gobernación de Santa Marta. Santa Marta: Banco de la República.

Salomón, M. (1980). Panorama de las principales corrientes de interpretación de la educación como fenómeno social. Perfiles educativos, 8(1), 3-24.

Sassoon, D. (2006). Cultura: el patrimonio común de los europeos. Barcelona: Grupo Planeta (GBS).
Troitiño, M. A. (1998). Patrimonio arquitectónico, cultura y territorio. Ciudades, 4. 95-104.

Valcárcel, J. (2018). El patrimonio territorial: el territorio como recurso cultural y económico. Ciudades, (04), 31-48. https://doi.org/10.24197/ciudades.04.1998.31-48

Yúdice, G. (2002). El recurso de la cultura. Barcelona: Gedisa. 